\title{
Risk Factors of Mechanical Ventilation in Premature Infants During Hospitalization
}

\section{Guang Yue \\ Jun Wang \\ Huaying Li \\ Biao Li \\ Rong Ju (D)}

Neonatal Department, Chengdu Women's and Children's Central Hospital, School of Medicine, University of Electronic Science and Technology of China, Chengdu, People's Republic of China
Correspondence: Rong Ju

Tel +86-13699003629

Email jurong@uestc.edu.cn
Background: The purpose of this study was to identify the risk factors for premature neonates requiring mechanical ventilation.

Methods: Premature neonates admitted to Chengdu Women's and Children's Central Hospital between July 2014 and December 2020 were retrospectively included in this study. Clinical and demographic factors were collated. Univariate and multivariate logistic regression analyses were conducted to identify the risk factors for premature infants requiring mechanical ventilation.

Results: A total of 1262 premature neonates participated in the study. Among them, 423 $(33.53 \%)$ neonates required mechanical ventilation, whereas 839 (66.48\%) neonates did not require mechanical ventilation. Multivariate logistic regression analysis determined that a lower Apgar score at $5 \mathrm{~min}(\mathrm{OR}=0.595,95 \% \mathrm{CI}$ : 0.472-0.74; $P<0.001)$, lower gestational age (very preterm) $(\mathrm{OR}=11.745,95 \% \mathrm{CI}: 4.362,31.619, P<0.001)$, lower systolic blood pressure $(\mathrm{OR}=0.864,95 \% \mathrm{CI}: 0.812-0.917, P=0.001)$, lower diastolic blood pressure $(\mathrm{OR}=0.894,95 \% \mathrm{CI}: 0.831-0.96, P=0.002)$, higher respiratory rate $(\mathrm{OR}=1.292$, 95\% CI: $1.238-1.355, P<0.001)$, increased C-reactive protein levels $(\mathrm{OR}=1.044,95 \% \mathrm{CI}$ : $1.003-1.086, P=0.036$ ), and presence of patent ductus arteriosus (OR $=2.174,95 \% \mathrm{CI}$ : $1.185-3.972, P=0.012$ ) were independently associated with an increased possibility of adopting mechanical ventilation in premature infants. ROC analysis demonstrated that the predicted power for premature neonates requiring mechanical ventilation was 0.855 (95\% CI: $0.808-0.902, P<0.001)$.

Conclusion: In conclusion, we determined that a lower Apgar score at $5 \mathrm{~min}$, lower gestational age, lower systolic blood pressure, lower diastolic blood pressure, higher respiratory rate, increased C-reactive protein levels and presence of patent ductus arteriosus were independently associated with an increased possibility of adopting mechanical ventilation in premature infants.

Keywords: mechanical ventilation, gestational age, patent ductus arteriosus, Apgar score, premature infants

\section{Introduction}

Preterm birth is one of the most challenging problems facing obstetric care and is closely associated with perinatal mortality and morbidity. Based on data collected from 184 countries, the average global preterm birth rate in 2018 was estimated to be $11.1 \%$, ranging from approximately $5 \%$ in northern Europe to $18 \%$ in subSaharan Africa. ${ }^{1}$ China accounts for $7.8 \%$ of all preterm births in the world with a mortality rate of preterm births at $7.1 \%$ of all live births. ${ }^{2}$ As the gestational age decreases among preterm births, a very significant increase in mortality begins to 
emerge. $^{3}$ It has been determined that an increased respiratory morbidity risk is more inextricably associated with preterm births than with term deliveries. ${ }^{4}$ Most preterm deaths occur among the earliest gestational age newborns with the causes of death including apnea, respiratory distress syndrome, necrotizing enterocolitis, intraventricular hemorrhage, infection, and asphyxia. ${ }^{2}$ Two of these are respiratory diseases. Respiratory problems are common after birth in preterm infants, especially extremely preterm infants $(<28$ weeks of gestation), and most of these infants require assisted ventilation until they are sufficiently mature and strong to breathe unassisted.

The evolution of neonatal respiratory support has been at the center of the advancements in neonatal-perinatal medicine and has facilitated the survival of infants that were previously considered to have no chance of survival. Mechanical ventilation is a life support treatment for patients who require support from a ventilator owing to severe illness in a hospital's intensive care unit (ICU). Although mechanical ventilation can also trigger complications with long-term implications, such as nosocomial infections, ${ }^{5,6}$ bronchopulmonary dysplasia, ${ }^{7-9}$ brain injury, ${ }^{10,11}$ retinopathy of prematurity $^{12}$ and poor neurodevelopmental outcome, ${ }^{8}$ some preterm infants require respiratory support at birth, which is usually lifesaving. The proportion of preterm infants requiring intubation and mechanical ventilation has decreased, and the most severely ill among them often require intubation and ventilation even after pulmonary surfactant treatment. As an increasing number of infants at the borderlines of viability receive full intensive care with the goal of intact survival, estimates of respiratory support are required to understand and predict resource utilization, as well as optimize the management of premature infants using mechanical ventilation. To promptly arrange and improve the respiratory status of infants, it is important to understand and determine the infants that require mechanical ventilation. However, the specific risk factors of mechanical ventilation in preterm infants remain unclear. The aim of this study was to explore the clinical features of premature infants that require mechanical ventilation and to identify the risk factors for premature infants requiring mechanical ventilation.

\section{Methods}

\section{Patients}

In this retrospective cohort study, we included premature infants admitted to Chengdu Women's and Children's
Central Hospital between July 2014 and December 2020. An infant born before 37 completed weeks of gestation is considered premature (full term is $37-42$ weeks). ${ }^{13}$ We excluded infants who died within $72 \mathrm{~h}$ of birth or those with major congenital malformations including congenital heart disease, digestive tract malformation and diaphragmatic hernia. Major congenital anomalies were defined as either life-threatening if untreated or potential triggers of significant neurodevelopmental disabilities, even if treated as described by Bassil et al. ${ }^{14}$ This study was approved by the Ethical Committee of Chengdu Women's and Children's Central Hospital. The informed consent requirement was waived because the study only involved the use of past clinical data. We have confirmed that the patient data was maintained with confidentiality, and that this study was conducted in accordance with the Declaration of Helsinki.

\section{Data Collection}

Clinical and demographic factors were collated, which included age, sex, height, birth weight, multiple births, in vitro fertilization (IVF), mode of delivery, Apgar score at the first minute, Apgar score at $5 \mathrm{~min}$, gestational age, previous admission to neonatal ICU (NICU), and patent ductus arteriosus (PDA) on the birth certificate. Respiratory rate, systolic blood pressure, diastolic blood pressure, heart rate, steroid therapy, blood product usage, enteral nutrition treatment, C-reactive protein (CRP), and anemia were collected on the first day of admission.

According to Queensland Clinical Guidelines, We divided studied population into three categories according to GA: <28wks'GA (extremely preterm), 28-31wks'GA (very preterm), and $32-37 \mathrm{wks}$ 'GA (moderate to late preterm). ${ }^{15}$

The Apgar score consists of five components (heart rate, respiratory effort, muscle tone, reflex irritability, and color), each with a value from 0 to 2 . Therefore, the total scores range from 0 to 10 , with higher scores indicating better physical condition.

Systolic blood pressure and diastolic blood pressure were measured indirectly using an oscillometric device (555; Corometrics Medical Systems, Wallingford, CT, USA) with the neonate awake, not crying and in the supine position after an appropriate-sized cuff was applied on the right arm. The blood pressure was measured three times in an interval of 2 minutes, and the average of the last two measurements was calculated for data analysis. ${ }^{16}$ 
Enteral nutrition treatment means the feeding amount reached $120 \mathrm{~mL} / \mathrm{kg}$ per day. While as the same time, removing peripherally inserted central catheter (PICC) was taken into consideration.

\section{Statistical Analysis}

Continuous variables were summarized as the median (interquartile range), and categorical variables were expressed as frequencies (percentages) based on variable characteristics and normal distribution. Comparisons of continuous and categorical variables were conducted using the Mann-Whitney $U$-test, Chi-squared test, or Fisher's exact test. The sample set was randomly divided into a training set ( $70 \%$ of the sample set) and testing set (30\% of the sample set). Logistic regression analysis was adopted to analyze the potential risk factors of premature neonates requiring mechanical ventilation. The variables with $P<0.1$ in the univariable logistic model were input into the multivariable logistic model in the training set. The backward selection method was adopted to identify factors associated with mechanical ventilation. Receiver operating characteristic curve (ROC) analysis was performed, and the area under the curve (AUC) was calculated in the testing set. Sensitivity and specificity were calculated based on the maximum Youden index. All statistical tests were two-sided, and statistical significance was set at $P<0.05$. The analyses were conducted using SPSS 22.0 (SPSS Inc., Chicago, IL).

\section{Results}

Comparison of Clinical Characteristics of Premature Neonates Requiring Mechanical Ventilation and Those Not Requiring Mechanical Ventilation

A total of 1262 premature neonates participated in the study (Table 1). Among them, 423 (33.53\%) neonates required mechanical ventilation, whereas 839 (66.48\%) neonates did not require mechanical ventilation. Age, birth weight, height, Apgar score at $1 \mathrm{~min}$, Apgar score at $5 \mathrm{~min}$, gestational age, systolic blood pressure, and diastolic blood pressure were significantly lower in premature neonates requiring mechanical ventilation than in premature neonates that did not require mechanical ventilation $(P<0.001)$. The respiratory rate, proportion of anemia, number of males, IVF pregnancy, enteral nutrition, CRP levels and PDA of preterm infants that required mechanical ventilation were higher than those that did not require mechanical ventilation $(P<0.05)$.

A total of $878(70 \%)$ premature neonates (293 requiring mechanical ventilation and 585 not requiring mechanical ventilation) were assigned to the training set while 384 $(30 \%)$ were assigned to the testing set (130 requiring mechanical ventilation and 254 not requiring mechanical ventilation). The baseline characteristics of premature neonates in the training and testing sets are presented in Table 2.

\section{Univariate and Multivariate Logistic Analyses of Risk Factors of Mechanical Ventilation in Premature Neonates}

Univariate logistic regression analysis indicated that age, sex, height, birth weight, Apgar score at the first minute, Apgar score at $5 \mathrm{~min}$, gestational age, systolic blood pressure, diastolic blood pressure, respiratory rate, CRP levels, anemia, and PDA were associated with mechanical ventilation in premature neonates (Table 3).

The final multivariable analysis included variables that were significantly associated with mechanical ventilation (Table 4). Finally, we determined that a lower Apgar score at $5 \min (\mathrm{OR}=0.595,95 \% \mathrm{CI}: 0.472-0.74 ; \mathrm{P}<0.001)$, lower gestational age (very preterm) $(\mathrm{OR}=11.745,95 \%$ CI: 4.362, 31.619, $\mathrm{P}<0.001)$, lower systolic blood pressure $(\mathrm{OR}=0.864,95 \% \mathrm{CI}: 0.812-0.917, \mathrm{P}=0.001)$, lower diastolic blood pressure (OR $=0.894,95 \%$ CI:0.831-0.96, $\mathrm{P}=0.002)$, higher respiratory rate $(\mathrm{OR}=1.292,95 \% \mathrm{CI}$ : 1.238-1.355, $\mathrm{P}<0.001)$, increased CRP levels $(\mathrm{OR}=1.044,95 \% \mathrm{CI}: 1.003-1.086, P=0.036)$, and presence of PDA (OR $=2.174,95 \%$ CI: $1.185-3.972, \mathrm{P}=$ 0.012 ) were independently associated with an increased risk mechanical ventilation in premature infants.

\section{Predictive Power Analysis of Premature Neonates Requiring Mechanical Ventilation}

A multivariate logistic regression model fitted to the training set was verified in the testing set. The ROC curve for the predicted risk from multivariate logistic regression of premature neonates requiring mechanical ventilation in the testing set is presented in Figure 1. The AUC, sensitivity, and specificity are 0.855 (95\% CI: $0.808,0.902, P<$ 0.001 ), 0.752 , and 0.870 , respectively (Table 5 and Figure 1). 
Table I Clinical Characteristics of Preterm Neonates

\begin{tabular}{|c|c|c|c|c|c|}
\hline & & $\begin{array}{l}\text { Requiring Mechanical } \\
\text { Ventilation }(n=423)\end{array}$ & $\begin{array}{l}\text { Not Requiring Mechanical } \\
\text { Ventilation }(n=839)\end{array}$ & Total $(n=1262)$ & $P$ value \\
\hline Age (days) & $\begin{array}{l}\text { Median } \\
\text { (Range) }\end{array}$ & $I(I, 6)$ & I $(1,24)$ & I $(1,24)$ & $<0.001$ \\
\hline Birth weight (kg) & Median (IQR) & $1.97(1.58,2.47)$ & $2.35(2.1,2.69)$ & $2.27(1.94,2.62)$ & $<0.001$ \\
\hline Length $(\mathrm{cm})$ & Median (IQR) & $43(40,47)$ & $46(44,48)$ & $46(43,48)$ & $<0.001$ \\
\hline Apgar score at I min & Median (IQR) & $8(7,9)$ & $9(9,10)$ & $9(8,10)$ & $<0.001$ \\
\hline Apgar score at $5 \mathrm{~min}$ & Median (IQR) & $9(9,10)$ & $10(10,10)$ & $10(9,10)$ & $<0.001$ \\
\hline Gestational age (week) & $\begin{array}{l}(0,28) \\
(28,32) \\
(32,37)\end{array}$ & $\begin{array}{l}13(100 \%) \\
124(93.9 \%) \\
286(25.6 \%)\end{array}$ & $\begin{array}{l}0(0 \%) \\
8(6.1 \%) \\
831(74.4 \%)\end{array}$ & $\begin{array}{l}13(100 \%) \\
132(100 \%) \\
1117(100 \%)\end{array}$ & $<0.001$ \\
\hline Respiratory rate (times/min) & Median (IQR) & $55(50,60)$ & $46(45,48)$ & $48(45,54)$ & $<0.001$ \\
\hline Systolic blood pressure $(\mathrm{mmHg})$ & Median (IQR) & $56(53,58)$ & $59(58,62)$ & $58(56,62)$ & $<0.001$ \\
\hline Diastolic blood pressure $(\mathrm{mmHg})$ & Median (IQR) & $34(31,36)$ & $36(34,38)$ & $35(32,38)$ & $<0.001$ \\
\hline Heart rate (BPM) & Median (IQR) & $138(132,142)$ & $140(135,140)$ & $140(135,140)$ & 0.069 \\
\hline C-reactive protein $(\mathrm{mg} / \mathrm{L})$ & Median (IQR) & $I(I, 5)$ & $I(I, 4)$ & $I(I, 4)$ & $<0.001$ \\
\hline Gender & $\begin{array}{l}\text { Female } \\
\text { Male }\end{array}$ & $\begin{array}{l}158(28.52 \%) \\
265(37.43 \%)\end{array}$ & $\begin{array}{l}396(71.48 \%) \\
443(62.57 \%)\end{array}$ & $\begin{array}{l}554(100 \%) \\
708(100 \%)\end{array}$ & 0.001 \\
\hline Multiple births & $\begin{array}{l}\text { No } \\
\text { Yes }\end{array}$ & $\begin{array}{l}215(36.94 \%) \\
108(30.25 \%)\end{array}$ & $\begin{array}{l}367(63.06 \%) \\
249(69.75 \%)\end{array}$ & $\begin{array}{l}582(100 \%) \\
357(100 \%)\end{array}$ & 0.036 \\
\hline In vitro fertilization & $\begin{array}{l}\text { No } \\
\text { Yes }\end{array}$ & $\begin{array}{l}410(33.12 \%) \\
13(54.17 \%)\end{array}$ & $\begin{array}{l}828(66.88 \%) \\
\text { II (45.83\%) }\end{array}$ & $\begin{array}{l}1238(100 \%) \\
24(100 \%)\end{array}$ & 0.03 \\
\hline Mode of delivery & $\begin{array}{l}\text { Spontaneous } \\
\text { labor } \\
\text { Cesarean } \\
\text { section }\end{array}$ & $\begin{array}{l}116(33.43 \%) \\
299(33.33 \%)\end{array}$ & $\begin{array}{l}23 \mathrm{I}(66.57 \%) \\
598(66.67 \%)\end{array}$ & $\begin{array}{l}347(100 \%) \\
897(100 \%)\end{array}$ & 0.974 \\
\hline Admitted to NICU & $\begin{array}{l}\text { No } \\
\text { Yes }\end{array}$ & $\begin{array}{l}416(33.44 \%) \\
7(38.89 \%)\end{array}$ & $\begin{array}{l}828(66.56 \%) \\
11(61.11 \%)\end{array}$ & $\begin{array}{l}1244(100 \%) \\
18(100 \%)\end{array}$ & 0.627 \\
\hline Enteral nutrition treatment & $\begin{array}{l}\text { No } \\
\text { Yes }\end{array}$ & $\begin{array}{l}379(32.76 \%) \\
44(41.9 \%)\end{array}$ & $\begin{array}{l}778(67.24 \%) \\
61(58.1 \%)\end{array}$ & $\begin{array}{l}1157(100 \%) \\
105(100 \%)\end{array}$ & $<0.001$ \\
\hline Anemia & $\begin{array}{l}\text { Yes } \\
\text { No }\end{array}$ & $\begin{array}{l}101 \text { (42.62\%) } \\
319(31.74 \%)\end{array}$ & $\begin{array}{l}136(57.38 \%) \\
686(68.26 \%)\end{array}$ & $\begin{array}{l}237(100 \%) \\
1005(100 \%)\end{array}$ & 0.002 \\
\hline Patent ductus arteriosus & $\begin{array}{l}\text { No } \\
\text { Yes }\end{array}$ & $\begin{array}{l}294(28.38 \%) \\
129(57.08 \%)\end{array}$ & $\begin{array}{l}742(71.62 \%) \\
97(42.92 \%)\end{array}$ & $\begin{array}{l}1036(100 \%) \\
226(100 \%)\end{array}$ & $<0.001$ \\
\hline Respiratory distress syndrome & $\begin{array}{l}\text { No } \\
\text { Yes }\end{array}$ & $\begin{array}{l}208(19.87 \%) \\
215(100 \%)\end{array}$ & $\begin{array}{l}839(80.13 \%) \\
0(0 \%)\end{array}$ & $\begin{array}{l}1047(100 \%) \\
215(100 \%)\end{array}$ & $<0.001$ \\
\hline
\end{tabular}


Table 2 Clinical Characteristics of Preterm Neonates in Training and Testing Sets

\begin{tabular}{|c|c|c|c|c|c|}
\hline & & \multicolumn{2}{|c|}{ Training Set $(n=878)$} & \multicolumn{2}{|c|}{ Testing Set $(n=384)$} \\
\hline & & $\begin{array}{l}\text { Requiring } \\
\text { Mechanical } \\
\text { Ventilation }(n=293)\end{array}$ & $\begin{array}{l}\text { Not Requiring } \\
\text { Mechanical Ventilation } \\
(n=585)\end{array}$ & $\begin{array}{l}\text { Requiring } \\
\text { Mechanical } \\
\text { Ventilation }(n=130)\end{array}$ & $\begin{array}{l}\text { Not Requiring } \\
\text { Mechanical Ventilation } \\
(n=254)\end{array}$ \\
\hline Age (days) & $\begin{array}{l}\text { Median } \\
\text { (Range) }\end{array}$ & $I(I, 6)$ & I $(I, 24)$ & $I(I, 4)$ & I $(I, 24)$ \\
\hline Birth weight (kg) & Median (IQR) & $1.9(1.47,2.4)$ & $2.34(2.09,2.7 I)$ & $2.13(1.78,2.6)$ & $2.36(2.13,2.65)$ \\
\hline Length $(\mathrm{cm})$ & Median (IQR) & $43(40,46)$ & $46(44,48)$ & $45(42,48)$ & $46(44,48)$ \\
\hline $\begin{array}{l}\text { Apgar score at I } \\
\min \end{array}$ & Median (IQR) & $8(7,9)$ & $9(9,10)$ & $9(8,9)$ & $9(9,10)$ \\
\hline $\begin{array}{l}\text { Apgar score at } 5 \\
\min \end{array}$ & Median (IQR) & $9(9,10)$ & $10(10,10)$ & $9(9,10)$ & $10(10,10)$ \\
\hline \multirow[t]{3}{*}{ Gestational age } & $(0,28)$ & $10(3.4 \%)$ & $0(0.0 \%)$ & $3(2.3 \%)$ & $0(0.0 \%)$ \\
\hline & $(28,32)$ & 97 (33.1\%) & $6(1.0 \%)$ & 27 (20.8\%) & $2(0.8 \%)$ \\
\hline & $(32,37)$ & $186(63.5 \%)$ & 579 (99.0\%) & $100(76.9 \%)$ & 252 (99.2\%) \\
\hline $\begin{array}{l}\text { Respiratory rate } \\
\text { (times/min) }\end{array}$ & Median (IQR) & $55(50,60)$ & $46(44,48)$ & $55(50,58)$ & $46(45,48)$ \\
\hline $\begin{array}{l}\text { Systolic blood } \\
\text { pressure }(\mathrm{mmHg})\end{array}$ & Median (IQR) & $56(53,58)$ & $59(58,62)$ & $58(54,60)$ & $58(58,62)$ \\
\hline $\begin{array}{l}\text { Diastolic blood } \\
\text { pressure }(\mathrm{mmHg})\end{array}$ & Median (IQR) & $33(31,36)$ & $36(34,38)$ & $35(32,38)$ & $36(34,38)$ \\
\hline Heart rate (BPM) & Median (IQR) & $138(132,142)$ & $140(135,140)$ & $136(130,140)$ & $140(135,140)$ \\
\hline $\begin{array}{l}\text { C-reactive } \\
\text { protein }(\mathrm{mg} / \mathrm{L})\end{array}$ & Median (IQR) & $I(I, 5)$ & $I(I, 4)$ & $I(I, 5)$ & $I(I, 4)$ \\
\hline \multirow[t]{2}{*}{ Gender } & Female & 108 (36.86\%) & $276(47.18 \%)$ & $50(38.46 \%)$ & 120 (47.24\%) \\
\hline & Male & 185 (63.14\%) & 309 (52.82\%) & $80(61.54 \%)$ & I 34 (52.76\%) \\
\hline \multirow[t]{2}{*}{ Multiple births } & No & $15 \mid$ (65.65\%) & 249 (59.29\%) & 64 (68.82\%) & 118 (60.2\%) \\
\hline & Yes & 79 (34.35\%) & |7| (40.7|\%) & 29 (31.18\%) & 78 (39.8\%) \\
\hline \multirow{2}{*}{$\begin{array}{l}\text { In vitro } \\
\text { fertilization }\end{array}$} & No & 284 (96.93\%) & $578(98.8 \%)$ & 126 (96.92\%) & 250 (98.43\%) \\
\hline & Yes & 9 (3.07\%) & 7 (1.2\%) & $4(3.08 \%)$ & $4(1.57 \%)$ \\
\hline \multirow[t]{2}{*}{ Mode of delivery } & $\begin{array}{l}\text { Spontaneous } \\
\text { labor }\end{array}$ & 86 (29.97\%) & $15 \mid(26.12 \%)$ & 30 (23.44\%) & 80 (31.87\%) \\
\hline & $\begin{array}{l}\text { Cesarean } \\
\text { section }\end{array}$ & 201 (70.03\%) & 427 (73.88\%) & 98 (76.56\%) & $17 \mid(68.13 \%)$ \\
\hline \multirow{2}{*}{$\begin{array}{l}\text { Admitted to } \\
\text { NICU }\end{array}$} & No & 288 (98.29\%) & 578 (98.8\%) & I 28 (98.46\%) & 250 (98.43\%) \\
\hline & Yes & 5 (I.7I\%) & 7 (1.2\%) & $2(1.54 \%)$ & $4(1.57 \%)$ \\
\hline \multirow{2}{*}{$\begin{array}{l}\text { Enteral nutrition } \\
\text { treatment }\end{array}$} & No & 265 (90.44\%) & 537 (91.79\%) & II 4 (87.69\%) & $24 \mathrm{I}(94.88 \%)$ \\
\hline & Yes & $28(9.56 \%)$ & 48 (8.21\%) & $16(12.31 \%)$ & $13(5.12 \%)$ \\
\hline
\end{tabular}


Table 2 (Continued).

\begin{tabular}{|l|l|l|l|l|l|}
\hline \multirow{2}{*}{} & & \multicolumn{2}{|c|}{ Training Set (n= 878) } & \multicolumn{2}{c|}{ Testing Set (n= 384) } \\
\cline { 3 - 6 } & & $\begin{array}{l}\text { Requiring } \\
\text { Mechanical } \\
\text { Ventilation }(\mathbf{n = 2 9 3 )}\end{array}$ & $\begin{array}{l}\text { Not Requiring } \\
\text { Mechanical Ventilation } \\
(\mathbf{n = 5 8 5})\end{array}$ & $\begin{array}{l}\text { Requiring } \\
\text { Mechanical } \\
\text { Ventilation }(\mathbf{n}=130)\end{array}$ & $\begin{array}{l}\text { Not Requiring } \\
\text { Mechanical Ventilation } \\
(\mathbf{n}=254)\end{array}$ \\
\hline \multirow{2}{*}{ Anemia } & Yes & $60(20.7 \%)$ & $87(15.2 \%)$ & $41(31.5 \%)$ & $49(19.6 \%)$ \\
\cline { 2 - 6 } & No & $230(79.3 \%)$ & $485(84.8 \%)$ & $89(68.5 \%)$ & $201(80.4 \%)$ \\
\hline \multirow{2}{*}{$\begin{array}{l}\text { Patent ductus } \\
\text { arteriosus }\end{array}$} & No & $200(68.3 \%)$ & $520(88.9 \%)$ & $94(72.3 \%)$ & $222(87.4 \%)$ \\
\cline { 2 - 6 } & Yes & $93(31.7 \%)$ & $65(11.1 \%)$ & $36(27.7 \%)$ & $32(12.6 \%)$ \\
\hline $\begin{array}{l}\text { Respiratory } \\
\text { distress syndrome }\end{array}$ & No & $138(47.1 \%)$ & $585(100 \%)$ & $60(46.2 \%)$ & $254(100 \%)$ \\
\cline { 2 - 6 } & Yes & $155(52.9 \%)$ & $0(0 \%)$ & $0(0 \%)$ \\
\hline
\end{tabular}

\section{Discussion}

In this retrospective study, we investigated the risk factors of mechanical ventilation in premature infants during hospitalization. We determined that a lower Apgar score at 5 min, lower gestational age (very preterm), lower systolic blood pressure, lower diastolic blood pressure, higher respiratory rate, increased CRP levels and presence of PDA were independently associated with an increased possibility of using mechanical ventilation in premature infants. ROC analysis verified that the predicted power of multivariate logistic regression for premature neonates requiring mechanical ventilation in the testing set was 0.855 .

Prematurity is conventionally considered to be associated with an increased risk of long-term morbidity towing to the immaturity of different systems, specifically the respiratory system. ${ }^{17,18} \mathrm{~A}$ study demonstrated that preterm births were more inextricably associated with an increased risk of respiratory morbidity than term deliveries. ${ }^{4}$ Premature neonates require mechanical ventilation for a certain duration during the newborn period, and more than two-thirds of those born at less than 29 weeks of gestation require mechanical ventilation. ${ }^{6}$ In this study, we determined that the median gestational age in premature neonates requiring mechanical ventilation was 33.9, compared to 35.7 in premature neonates not requiring mechanical ventilation. Decreased gestational age was considered an independent risk factor associated with premature neonates requiring mechanical ventilation. A cross-sectional study by Moreira et al in Brazil determined that $13.1 \%$ of neonates with a gestational age greater than 34 weeks required supplementary oxygen. ${ }^{19}$ In this study, we determined that $33.53 \%$ of neonates required mechanical ventilation because we included extremely preterm infants ( $<28$ weeks of gestation). Sousa et $\mathrm{al}^{20}$ and Moreira et $\mathrm{al}^{19}$ inferred that preterm infants (with gestational age $<36$ weeks) exhibited an increased need for positive-pressure ventilation and supplemental oxygen than term infants, which was consistent with our study results, in which a lower gestational age was associated with an increased possibility of using mechanical ventilation in premature infants. The younger the gestational age of premature infants, the less mature the lung development and respiratory physiological function. At approximately the time of earliest extrauterine viability at 23 to 24 weeks of gestation, the distal lung parenchyma transitions from the canalicular to the saccular stage of lung development. Alveoli begin to appear after 32 weeks of gestation, and approximately $30 \%$ of the adult number of alveoli are formed by term. ${ }^{21,22}$ In preterm infants, the division of the alveolar saccules and ducts into true alveoli is yet to occur, and proliferation of the capillary network is still incomplete; therefore, the lungs are significantly unprepared for their essential role in gas exchange after birth. ${ }^{22}$ In addition, owing to the immature respiratory central regulatory function, respiratory dysfunction, or secondary to different underlying diseases, premature infants often experience apnea and respiratory failure. These factors significantly increase the possibility of premature infants receiving respiratory support treatment. Previous studies have determined that premature infants exhibited a statistically higher proportion of respiratory distress syndrome, transient tachypnea 
Table 3 Univariate Logistic Analysis of Risk Factors of Mechanical Ventilation in Premature Neonates

\begin{tabular}{|c|c|c|c|}
\hline Variables & OR & OR $95 \% \mathrm{Cl}$ & $P$ value \\
\hline Gender (Male vs Female) & 1.53 & $(1.148-2.04)$ & 0.004 \\
\hline Age & 0.507 & $(0.38 \mathrm{I}-0.677)$ & $<0.001$ \\
\hline Birth weight & 0.15 & $(0.106-0.211)$ & $<0.001$ \\
\hline Length & 0.758 & $(0.722-0.797)$ & $<0.001$ \\
\hline Multiple births (Yes vs No) & 0.762 & $(0.545-1.065)$ & 0.111 \\
\hline In vitro fertilization (Yes vs No) & 2.615 & $(0.964-7.094)$ & 0.059 \\
\hline Mode of delivery (Spontaneous labor vs Cesarean section) & 0.827 & $(0.604-1.131)$ & 0.233 \\
\hline Apgar score at I min & 0.605 & $(0.542-0.675)$ & $<0.001$ \\
\hline Apgar score at $5 \mathrm{~min}$ & 0.397 & $(0.322-0.489)$ & $<0.001$ \\
\hline Gestational age ( $<28$ weeks vs $32 \sim 37$ weeks) & 1 & 1 & $>0.99$ \\
\hline Gestational age (28 3I weeks vs 32 37 weeks) & 50.238 & $(21.668-116.477)$ & $<0.001$ \\
\hline Admitted to NICU (Yes vs No) & 1.434 & $(0.45 \mathrm{I}-4.556)$ & 0.542 \\
\hline Respiratory rate & 1.263 & $(1.222-1.306)$ & $<0.001$ \\
\hline Systolic blood pressure & 0.799 & $(0.767-0.832)$ & $<0.001$ \\
\hline Diastolic blood pressure & 0.811 & $(0.777-0.847)$ & $<0.001$ \\
\hline Heart rate & 0.996 & $(0.98 I-1.012)$ & 0.65 \\
\hline Enteral nutrition treatment (Yes vs No) & 1.182 & $(0.725-1.927)$ & 0.502 \\
\hline C-reactive protein & 1.05 & $(1.019-1.082)$ & 0.002 \\
\hline Anemia (Yes vs No) & 0.688 & $(0.479-0.993)$ & 0.044 \\
\hline Patent ductus arteriosus (Yes vs No) & 3.72 & $(2.6 I I-5.33)$ & $<0.001$ \\
\hline
\end{tabular}

Table 4 Multivariate Logistic Analysis of Risk Factors of Mechanical Ventilation in Premature Neonates

\begin{tabular}{|l|l|l|l|}
\hline Variables & OR & OR (95\% CI) & $P$ value \\
\hline Apgar score at 5 min & 0.595 & $(0.472-0.74)$ & $<0.00 I$ \\
\hline Gestational age (<28 weeks vs 32 37 weeks) & 1 & 1 & $>0.999$ \\
\hline Gestational age (28 3I weeks vs 32 37 weeks) & 11.745 & $(4.362-31.619)$ & $<0.00 I$ \\
\hline Respiratory rate & 1.292 & $(1.238-1.355)$ & $<0.001$ \\
\hline Systolic blood pressure & 0.864 & $(0.812-0.917)$ & 0.001 \\
\hline Diastolic blood pressure & 0.894 & $(0.831-0.96)$ & 0.002 \\
\hline Patent ductus arteriosus & 2.174 & $(I .185-3.972)$ & 0.012 \\
\hline C-reactive protein & 1.044 & $(I .003-1.086)$ & 0.036 \\
\hline
\end{tabular}




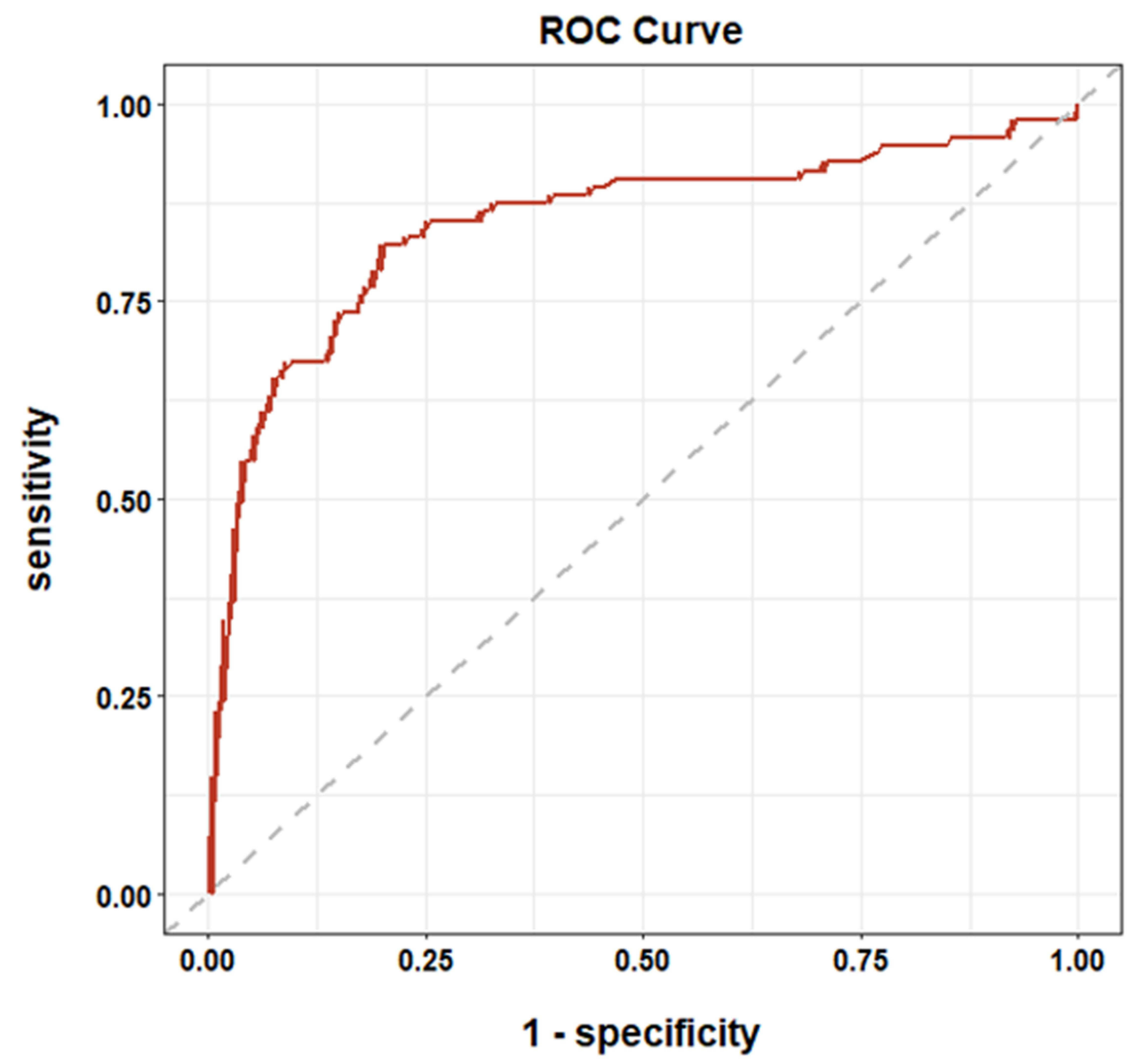

Figure I ROC analysis of premature infants requiring mechanical ventilation.

of the newborn, pneumonia, respiratory failure, and standard than term neonates. ${ }^{4,23,24}$ Although the development trend of neonatal ICUs is dedicated to avoiding mechanical ventilation substantially, mechanical ventilation remains a necessary means of supporting premature infants with lung disease, especially extremely preterm infants.

Interestingly, we determined that a lower Apgar score was another independent risk factor associated with premature neonates requiring mechanical ventilation. Dalili et al inferred that a low 5-minute combined Apgar score remained significantly associated with the requirement for mechanical ventilation, ${ }^{25}$ which is consistent with our

Table 5 Prediction Values of Mechanical Ventilation in Premature Neonates in Testing Set

\begin{tabular}{|l|l|l|l|}
\hline & AUC $(\mathbf{9 5} \% \mathbf{C l})$ & Sensitivity & Specificity \\
\hline $\begin{array}{l}\text { Logistic } \\
\text { regression }\end{array}$ & $0.855(0.808,0.902)$ & 0.752 & 0.870 \\
\hline
\end{tabular}

study. Among five components consisting the Apgar score, two stems (color and respiratory effort) are linked to the respiratory status, a low Apgar score is associated with a higher risk of immediate respiratory morbidity. ${ }^{26}$ A low Apgar score increases the chances of several longterm respiratory-related morbidities, independent of gestational age and other obstetrical circumstances. ${ }^{26} \mathrm{~A}$ low Apgar score in preterm infants may indicate biological immaturity. ${ }^{27}$ Therefore, respiratory morbidity, at least in the short term, is expected to be more prevalent among newborns with low Apgar scores. Therefore, clinicians should pay more attention to the respiratory status of premature infants with low Apgar scores.

PDA is another independent high-risk factor associated with premature neonates requiring mechanical ventilation. PDA is a relatively common challenged encountered by preterm infants, with an incidence showing an inverse correlation with gestational age. ${ }^{28,29}$ In preterm infants, however, PDA closure is delayed, remaining open at 7 days of age in approximately $2 \%$ of infants born at 30 
37 weeks of gestation, with $65 \%$ of them born at $25-28$ weeks of gestation, and $87 \%$ of them at 24 weeks of gestation. $^{30} \mathrm{~A}$ persistent left-to-right shunt through a PDA increases the rate of hydrostatic fluid filtration into the lung's interstitium, impairs pulmonary mechanics, produces an acute deterioration in lung function, ${ }^{31}$ and prolongs the need for mechanical ventilation. ${ }^{32}$ Studies have demonstrated that preterm infants treated with PDA ligation achieved earlier successful extubation..$^{33,34}$ These findings suggest that PDA can significantly trigger respiratory insufficiency. Premature infants with PDA may require mechanical ventilation; therefore, physicians should pay more attention to their respiratory function.

Lower blood pressure was a minor risk factor associated with an increased possibility of using mechanical ventilation in premature infants. Infants with birth asphyxia, as well as ventilated infants, had lower systolic and diastolic blood pressures than other infants without these conditions. ${ }^{35-37}$ Premature infants with low blood pressure have weak organ systems, including the respiratory system. Previous studies reported that the blood pressure of premature infants is lower than that of term infants at birth; ${ }^{37-40}$ however, blood pressure increased steadily in the first week of life. ${ }^{41}$ Therefore, the infants with low blood pressure were younger and had immature physiological functions; hence, the possibility of requiring mechanical ventilation increases.

Increased CRP levels were weakly associated with an increased possibility of using mechanical ventilation in premature infants. Previous studies have determined that increased CRP levels could be a useful marker of critical illness like respiratory distress in neonates and coronavirus disease 2019 (COVID-19) in children. ${ }^{42,43}$ And another study found that increased CRP levels were associated with a decrease in ventilator-free days in children. ${ }^{44}$ Herold et al found that higher CRP levels could increase the need of mechanical ventilation in COVID-19 patients. ${ }^{45}$ However, similar studies in preterm infants were no found. A prospective research with large numbers should be performed in future to find real relationship between CRP levels and mechanical ventilation in preterm infants.

The retrospective nature of this study is a significant limitation. On the one hand, it enabled us to analyze a large cohort of newborns, but on the other hand, it exhibits all the inherent weaknesses of a retrospective study. As with any retrospective review, causality cannot be confirmed, and only correlations can be inferred. Future prospective research could address some of these challenges. Second, the training and validation data adopted in this study were obtained from a single center. Because we did not include an external validation cohort in this study, the real values of the prediction models require further validated in future studies. Also, we could not evaluate other ante natal factors' associations with need of mechanical ventilation, like IUGR occurrence and steroids therapy. Since we only adopted data from a single center while many subjects taken prenatal examination in other hospital, complete data of these factors were not available. Finally, for some data like blood gases which recorded in papers instead of electronic medical record system, we could not adopt these indicators into our study because it is difficult to input the data into system considering the inconsistent record standards.

In conclusion, our study presents clinical and demographic information on premature neonates requiring mechanical ventilation. Ultimately, we determined that a lower Apgar score at $5 \mathrm{~min}$, lower gestational age (very preterm), lower systolic blood pressure, lower diastolic blood pressure, higher respiratory rate, increased CRP levels and presence of PDA were independently associated with an increased possibility of using mechanical ventilation in premature infants. These results could be helpful for clinicians to predict who could require mechanical ventilation and comprehensively assess the necessity of utilization of mechanical ventilation. In this way, it could be possible to avoid the damage of unnecessary use of mechanical ventilation.

\section{Acknowledgments}

We wish to thank Shanghai Synyi Medical Technology Co., Ltd. for providing a data analysis and statistical platform.

\section{Funding}

This study was approved by the Health Commission of Sichuan Province (No. 18PJ046) and Chengdu Science and Technology Bureau (No. 2016-HM01-00324-SF).

\section{Disclosure}

The authors declare no conflicts of interest for this work and that there are no competing interests associated with the manuscript.

\section{References}

1. World Health Organization. Preterm birth; 2018. Available from: https://www.who.int/news-room/fact-sheets/detail/preterm-birth. Accessed 19 February 2018. 
2. Harrison MS, Goldenberg RL. Global burden of prematurity. Semin Fetal Neonatal Med. 2016;21(2):74-79. doi:10.1016/j. siny.2015.12.007

3. Principi N, Di Pietro GM, Esposito S. Bronchopulmonary dysplasia: clinical aspects and preventive and therapeutic strategies. $J$ Transl Med. 2018;16(1):36. doi:10.1186/s12967-018-1417-7

4. Hibbard JU, Wilkins I, Sun L, et al. Respiratory morbidity in late preterm births. JAMA. 2010;304(4):419-425.

5. Kawagoe JY, Segre CA, Pereira CR, Cardoso MF, Silva CV, Fukushima JT. Risk factors for nosocomial infections in critically ill newborns: a 5-year prospective cohort study. Am J Infect Control. 2001;29(2):109-114. doi:10.1067/mic.2001.114162

6. Stoll BJ, Hansen NI, Bell EF, et al. Neonatal outcomes of extremely preterm infants from the NICHD Neonatal Research Network. Pediatrics. 2010;126(3):443-456. doi:10.1542/peds.2009-2959

7. Thébaud B, Goss KN, Laughon M, et al. Bronchopulmonary dysplasia. Nat Rev Dis Primers. 2019;5(1):78.

8. Walsh MC, Morris BH, Wrage LA, et al. Extremely low birthweight neonates with protracted ventilation: mortality and 18 -month neurodevelopmental outcomes. $J$ Pediatr. 2005;146(6):798-804. doi:10.1016/j.jpeds.2005.01.047

9. Tracy MK, Berkelhamer SK. Bronchopulmonary dysplasia and pulmonary outcomes of prematurity. Pediatr Ann. 2019;48(4):e148e153. doi:10.3928/19382359-20190325-03

10. Ophelders D, Gussenhoven R, Klein L, et al. Preterm brain injury, antenatal triggers, and therapeutics: timing is key. Cells. 2020;9 (8):1871. doi:10.3390/cells9081871

11. Cannavo L, Rulli I, Falsaperla R, Corsello G, Gitto E. Ventilation, oxidative stress and risk of brain injury in preterm newborn. Ital $J$ Pediatr. 2020;46(1):100. doi:10.1186/s13052-020-00852-1

12. Taner A, Tekle S, Hothorn T, Adams M, Bassler D, Gerth-Kahlert C. Higher incidence of retinopathy of prematurity in extremely preterm infants associated with improved survival rates. Acta Paediatr. 2020;109(10):2033-2039. doi:10.1111/apa.15197

13. Tucker J, McGuire W. Epidemiology of preterm birth. BMJ. 2004;329(7467):675-678. doi:10.1136/bmj.329.7467.675

14. Bassil KL, Collier S, Mirea L, et al. Association between congenital anomalies and area-level deprivation among infants in neonatal intensive care units. Am J Perinatol. 2013;30(3):225-232.

15. National Collaborating Centre for Women's and Children's Health (UK). Preterm Labour and Birth: Full guideline. London: National Institute for Health and Care Excellence (UK); 2015.

16. Pejovic B, Peco-Antic A, Marinkovic-Eric J. Blood pressure in non-critically ill preterm and full-term neonates. Pediatr Nephrol. 2007;22(2):249-257. doi:10.1007/s00467-006-0311-3

17. Islam JY, Keller RL, Aschner JL, Hartert TV, Moore PE. Understanding the short- and long-term respiratory outcomes of prematurity and bronchopulmonary dysplasia. Am J Respir Crit Care Med. 2015;192(2):134-156. doi:10.1164/rccm.201412-2142PP

18. Manuck TA, Rice MM, Bailit JL, et al. Preterm neonatal morbidity and mortality by gestational age: a contemporary cohort. Am J Obstet Gynecol. 2016;215(1):103.e101-103.e114. doi:10.1016/j. ajog.2016.01.004

19. Moreira ME, Pereira AP, Gomes Junior SC, et al. Factors associated with the use of supplemental oxygen or positive pressure ventilation in the delivery room, in infants born with a gestational age $\geq 34$ weeks. Reprod Health. 2016;13(Suppl 3):116. doi:10.1186/s12978016-0235-8

20. Sousa JR, Leite ÁJ, Sanudo A, Guinsburg R. Factors associated with the need for ventilation at birth of neonates weighing $\geq 2500 \mathrm{~g}$. Clinics (Sao Paulo). 2016;71(7):381-386. doi:10.6061/clinics/ 2016(07)05

21. Jobe AH. An unknown: lung growth and development after very preterm birth. Am J Respir Crit Care Med. 2002;166 (12):1529-1530. doi:10.1164/rccm.2209012
22. Smith LJ, McKay KO, van Asperen PP, Selvadurai H, Fitzgerald DA. Normal development of the lung and premature birth. Paediatr Respir Rev. 2010;11(3):135-142. doi:10.1016/j.prrv.2009.12.006

23. Tsai ML, Lien R, Chiang MC, et al. Prevalence and morbidity of late preterm infants: current status in a medical center of Northern Taiwan. Pediatr Neonatol. 2012;53(3):171-177. doi:10.1016/j. pedneo.2012.04.003

24. Bond S. Late preterm newborns suffer significant respiratory morbidity. J Midwifery Womens Health. 2011;56(1):83-84. doi:10.1111/j.1542-2011.2010.00011_3.x

25. Dalili H, Sheikh M, Hardani AK, Nili F, Shariat M, Nayeri F. Comparison of the combined versus conventional Apgar scores in predicting adverse neonatal outcomes. PLoS One. 2016;11(2): e0149464. doi:10.1371/journal.pone.0149464

26. Ernest E, Wainstock T, Sheiner E, Segal I, Landau D, Walfisch A. Apgar score and long-term respiratory morbidity of the offspring: a population-based cohort study with up to 18 years of follow-up. Eur J Pediatr. 2019;178(3):403-411. doi:10.1007/s00431-018-03311-6

27. Cnattingius S, Johansson S, Razaz N. Apgar score and risk of neonatal death among preterm infants. $N$ Engl $J$ Med. 2020;383 (1):49-57. doi:10.1056/NEJMoa1915075

28. McNamara PJ, Sehgal A. Towards rational management of the patent ductus arteriosus: the need for disease staging. Arch Dis Child Fetal Neonatal Ed. 2007;92(6):F424-F427. doi:10.1136/adc.2007.118117

29. Clyman RI, Couto J, Murphy GM. Patent ductus arteriosus: are current neonatal treatment options better or worse than no treatment at all? Semin Perinatol. 2012;36(2):123-129. doi:10.1053/j. semperi.2011.09.022

30. Benitz WE. Patent ductus arteriosus in preterm infants. Pediatrics. 2016;137(1):e20153730. doi:10.1542/peds.2015-3730

31. Bancalari E. Patent ductus arteriosus and short- and long-term respiratory outcomes. Am J Perinatol. 2016;33(11):1055-1057. doi:10.1055/s-0036-1586112

32. Clyman RI. Patent ductus arteriosus, its treatments, and the risks of pulmonary morbidity. Semin Perinatol. 2018;42(4):235-242. doi:10.1053/j.semperi.2018.05.006

33. Shi X, Hua Y, Li Y. Transcatheter closure of patent ductus arteriosus in preterm ventilation-dependent neonates: a case series report. Medicine (Baltimore). 2020;99(47):e22528. doi:10.1097/ MD.0000000000022528

34. Krishnappa S, Shah PS, Jain A, Resende MHF, McNamara PJ, Weisz DE. Predictors of early extubation after patent ductus arteriosus ligation among infants born extremely preterm dependent on mechanical ventilation. $J$ Pediatr. 2019;214:222-226 e223. doi:10.1016/j.jpeds.2019.06.027

35. Göpel W, Müller M, Rabe H, et al. Genetic background of high blood pressure is associated with reduced mortality in premature neonates. Arch Dis Child Fetal Neonatal Ed. 2020;105(2):184-189. doi:10.1136/archdischild-2019-317131

36. Pas AT, Sobotka K, Hooper SB. Novel approaches to neonatal resuscitation and the impact on birth asphyxia. Clin Perinatol. 2016;43 (3):455-467. doi:10.1016/j.clp.2016.04.005

37. Faust K, Härtel C, Preuß M, et al. Short-term outcome of very-lowbirthweight infants with arterial hypotension in the first $24 \mathrm{~h}$ of life. Archiv Dis Childhood Fetal Neonatal Edition. 2015;100(5):F388F392. doi:10.1136/archdischild-2014-306483

38. Du BW, Wang J, Sun K. [Characteristics of blood pressure and the mechanisms of increased risk of hypertension in premature infants]. Zhonghua Er Ke Za Zhi. 2020;58(2):155-158. Chinese.

39. Samanta M, Mondal R, Ray S, et al. Blood pressure variation with gestational age and birth weight in Indian newborn. $J$ Trop Pediatr. 2015;61(3):197-205. doi:10.1093/tropej/fmv019

40. Logan JW, O'Shea TM, Allred EN, et al. Early postnatal hypotension and developmental delay at 24 months of age among extremely low gestational age newborns. Archiv Dis Childhood Fetal Neonatal Edition. 2011;96(5):F321-F328. doi:10.1136/adc.2010.183335 
41. Nascimento M, Xavier C, Goulart E. Arterial blood pressure of term newborns during the first week of life. Braz J Med Biol Res. 2002;35:905-911. doi:10.1590/S0100-879X2002000800007

42. Bhumbra S, Malin S, Kirkpatrick L, Khaitan A, Enane LA. Clinical features of critical Coronavirus disease 2019 in children. Pediatr Crit Care Med. 2020;21:e948-e953. doi:10.1097/PCC.0000000000002511

43. Rego M, Martinez FE, Elias J, Mussi-Pinhata MM. Diagnostic value of interleukin-6 and C-reactive protein on early onset bacterial infection in preterm neonates with respiratory distress. $J$ Perinat Med. 2010;38(5):527-533. doi:10.1515/jpm.2010.071
44. Bruijn M, Jansen EM, Klapwijk T, Lee J. Association between C-reactive protein levels and outcome in acute lung injury in children. Eur J Pediatr. 2013;172(8):1105-1110. doi:10.1007/s00431-013-2006-7

45. Herold T, Jurinovic V, Arnreich C, et al. Elevated levels of IL-6 and CRP predict the need for mechanical ventilation in COVID-19. J Allerg Clin Immunol. 2020;146(1):128-136. e124. doi:10.1016/j. jaci.2020.05.008

\section{Publish your work in this journal}

Therapeutics and Clinical Risk Management is an international, peerreviewed journal of clinical therapeutics and risk management, focusing on concise rapid reporting of clinical studies in all therapeutic areas, outcomes, safety, and programs for the effective, safe, and sustained use of medicines. This journal is indexed on PubMed Central, CAS,
EMBase, Scopus and the Elsevier Bibliographic databases. The manuscript management system is completely online and includes a very quick and fair peer-review system, which is all easy to use. Visit http://www.dovepress.com/testimonials.php to read real quotes from published authors. 